\title{
Comparative analysis and diagnostic accuracy of the cervical flexion-rotation test
}

\author{
Toby M. Hall $\cdot$ Kathy Briffa $\cdot$ Diana Hopper • \\ Kim Robinson
}

Received: 15 March 2010/Accepted: 26 April 2010/Published online: 28 May 2010

(C) Springer-Verlag 2010

\begin{abstract}
The aim of this study was to compare the findings of the cervical flexion-rotation test (FRT) between subjects with probable cervicogenic headache (CGH), migraine without aura (Migraine), and multiple headache forms (MHF). An additional aim was to identify the diagnostic accuracy of the FRT in CGH evaluation. Sixty subjects were evaluated: 20 with CGH, 20 with Migraine, and 20 with MHF. Subject and headache symptoms were evaluated by questionnaire. A single-blind examiner conducted the FRT, reporting the test state (positive or negative) before measuring range of motion using a goniometer. The average range of unilateral rotation to the most restricted side was $25^{\circ}, 42^{\circ}$ and $35^{\circ}$ for groups $\mathrm{CGH}$, Migraine and MHF, respectively. The difference between groups was significant $(P<0.001)$. Range of rotation was significantly reduced in the $\mathrm{CGH}$ group when compared to groups Migraine $(P<0.001)$ and MHF $(P=0.001)$, with an additional smaller significant difference between groups Migraine and MHF $(P=0.039)$. A receiver operating curve revealed that an experienced examiner using the FRT was able to make the correct diagnosis $85 \%$ of the time $(P<0.001)$, with a positive cut-off value of $30^{\circ}$. Multivariate regression analysis revealed that $44 \%$ of the variance in FRT range of motion was explained by the presence of two variables: neck movement or positions provoke headache, and neck symptoms precede headache, but not by other factors associated with migraine. These findings provide further evidence supporting the clinical utility of the FRT in CGH evaluation.
\end{abstract}

T. M. Hall $(\bowtie) \cdot$ K. Briffa · D. Hopper · K. Robinson School of Physiotherapy, Curtin Innovation Health Research Institute, Curtin University of Technology, Bentley, Perth, Western Australia

e-mail: halltm@netspace.net.au
Keywords Cervicogenic headache $\cdot$ Migraine . Neck $\cdot$ Sensitivity and specificity $\cdot$ Range of motion

\section{Introduction}

Headache is among the most prevalent of pain disorders, affecting the majority of the population [1], disturbing both quality of life and work productivity [2,3].

Migraine is one of the common primary headaches [4], the underlying mechanisms of which are poorly understood but are believed to involve abnormal brain function [5] including functional cortical hyperexcitability arising from among other things, reduced inhibition from the cortex [6]. In contrast cervicogenic headache $(\mathrm{CGH})$ is a subgroup of secondary headache, stemming from a disorder of the cervical spine [7]. The mechanisms underlying CGH [8] are based on the concept of convergence of afferent information from musculoskeletal structures in the upper three cervical segments with trigeminal afferents in the trigeminocervical nucleus (TCN). Hence input via sensory afferents from any of the upper cervical nerve roots is mistakenly perceived as pain in the head.

Differentiating different headache forms is principally based on the history and presenting symptoms, together with the clinical physical examination [9]. For example, migraine without aura is the most common sub-type of migraine, lasting a fixed time period of 24-72 h. Diagnostic characteristics include unilateral location, pulsating quality, moderate to severe intensity, aggravated by activity, and associated with nausea, photophobia or phonophobia [10]. However, a number of these features are also associated with CGH [11] as is the presence of neck pain [12]. As a result, one of the common diagnostic challenges in headache evaluation is to distinguish CGH from migraine [13]. Indeed studies have shown that an incorrect headache diagnosis may occur in more than $50 \%$ of 
cases [14]. Since migraine and CGH have very different underlying pathological mechanisms, it is very important to correctly classify the headache disorder so that treatment can be directed appropriately. For example, physiotherapy has been found to be effective for CGH [15, 16] but not for migraine [17].

Up to $74 \%$ of individuals with frequent intermittent headache and migraine report accompanying neck pain $[12,13$, 18], but this does not necessarily indicate dysfunction in the cervical spine. Associated neck symptoms during headache may simply suggest spread and referral of pain via trigeminocervical interaction, resulting in hyperalgesia and allodynia [12]. Consequently, physical examination of the neck is a critical component of CGH diagnosis [19], particularly where subjective characteristics are not clearly indicative of any one headache form or where multiple headache forms (MHF) co-exist. As CGH predominantly arises from musculoskeletal dysfunction in the upper three cervical segments [8], physical examination of the upper cervical spine is particularly important. Recently it has been shown that collectively, cervical movement impairment, in association with palpable upper cervical joint dysfunction and impairment in craniocervical muscle control, has $100 \%$ sensitivity and $94 \%$ specificity to identify CGH from migraine [20].

The flexion-rotation test (FRT) has shown promise as a valid and reliable test of upper cervical movement impairment associated with CGH [21-24]. Movement during this test occurs predominantly at $\mathrm{C} 1 / 2$, as a result of maximally flexing the cervical spine, pre-emptively constraining movement at all other levels [25]. We have previously demonstrated that the FRT has high levels of accuracy in CGH diagnosis [24], but the comparison group in that study were asymptomatic controls and a relatively "pure" form of migraine with aura, which is easily defined. To date no studies have investigated whether the FRT is reduced in mobility in subjects with Migraine or in subjects with subjective features suggesting MHF. If subjects with $\mathrm{CGH}$ have significantly greater impairment on the FRT than subjects with Migraine or with MHF, then this further validates the utility of the FRT in CGH evaluation.

The main purpose of this study was to compare FRT mobility between three groups: Migraine, $\mathrm{CGH}$, and MHF. Secondary purposes were to examine the diagnostic accuracy of the FRT in $\mathrm{CGH}$ diagnosis and to determine the most significant predictors of range of motion during the FRT. This knowledge has the potential to improve the clinical utility of the FRT.

\section{Methods}

A single-blind comparative measurement study design was used to determine whether range recorded during the FRT and examiner interpretation of the FRT differs between subjects with MHF and those with Migraine and CGH. The Curtin University Human Research Ethics Committee granted approval for this study, which was conducted in accordance with the ethical standards laid down in the 1964 Declaration of Helsinki. Subjects gave written informed consent prior to the study commencement and were able to withdraw from the study at any time.

\section{Subjects}

Sample size estimate was based on data collected from previous studies [24, 26]. Based on a single measurement of the FRT, with a standard deviation of $11^{\circ}$ for range of motion, and a calculated effect size of 0.4 [27, 28], a priori power analysis determined that a total sample size of 60 subjects (20 in each group) was required to obtain a statistical power of 0.80 with alpha set at 0.05 .

Subjects were recruited in a manner of convenience through advertisements placed in local newspapers and through Curtin University website. Volunteers were screened for eligibility into three groups: Migraine, $\mathrm{CGH}$ or MHF. For all subjects headache frequency was required to be at least once per week for more than the previous 3 months. Migraine was defined according to the IHS diagnostic criteria [10], CGH was defined according to the criteria proposed by Sjaastad [11] and further evaluated by Antonaci [29], with the exception of diagnostic anaesthetic blocks. These criteria were intermittent, side dominant headache of moderate intensity, without side shift. The headache was required to be preceded by ipsilateral neck pain and precipitated or aggravated by neck movement or posture, thereby fulfilling 5 of 7 criteria outlined by Antonaci [29] as indicative of "probable" CGH. Subjects in either the migraine or CGH group were excluded if they also met the IHS criteria for other headache forms. Subjects who reported two or more distinct forms of headache were defined as MHF. In all three groups subjects were excluded if they were unable to tolerate the FRT (none were excluded in this way) or if they had received physical treatment to their neck from a physiotherapist or other health professional in the previous 4 weeks. In addition, to account for the potential for a concurrent cervical spine disorder in the Migraine group, subjects were excluded if they had interictal episodes of neck pain or a known cervical spine disorder.

Respondents to advertisements $(n=115)$ were screened by either telephone or face-to-face interview with 55 rejected, according to the inclusion and exclusion criteria. Accordingly 20 subjects with Migraine (12 female, mean age 30 years, range 18-59, SD 6.5), 20 subjects with $\mathrm{CGH}$ (11 female, mean age 35 years, range 18-61, SD 10.9) and 
Table 1 Characteristics of the subjects in groups Migraine, CGH and MHF

\begin{tabular}{|c|c|c|c|}
\hline \multirow[t]{2}{*}{ Variable } & \multicolumn{3}{|l|}{ Headache group } \\
\hline & $\mathrm{CGH}(n=20)$ & Migraine $(n=20)$ & $\operatorname{MHF}(n=20)$ \\
\hline \multicolumn{4}{|l|}{ History of headache (years) } \\
\hline Mean (SD) & $4.8(2.8)$ & $9.1(4.8)$ & $5.7(3.9)$ \\
\hline Range & $0.5-11.0$ & $4.0-20.0$ & $0.5-14$ \\
\hline \multicolumn{4}{|l|}{ Index of headache severity $(/ 100)$} \\
\hline Mean (SD) & $56.3(10.8)$ & $54.1(14.1)$ & $47.9(11.4)$ \\
\hline Range & $35.0-81.0$ & $31.3-81.6$ & $31.3-71.0$ \\
\hline \multicolumn{4}{|l|}{ Dominant side of headache } \\
\hline Left & 8 & 4 & 5 \\
\hline Right & 12 & 7 & 11 \\
\hline Bilateral & 0 & 9 & 4 \\
\hline Positive flexion-rotation test & 14 & 5 & 8 \\
\hline Pulsating headache & 4 & 12 & 7 \\
\hline Photophobia & 5 & 16 & 8 \\
\hline Phonophobia & 4 & 15 & 9 \\
\hline Nausea & 5 & 16 & 9 \\
\hline Neck positions/movement provoke headache & 20 & 3 & 8 \\
\hline Neck symptoms precede headache & 17 & 4 & 10 \\
\hline Anti-migraine medication helps & 1 & 13 & 5 \\
\hline Forward bend increases headache & 6 & 13 & 9 \\
\hline Exertion increases headache & 6 & 16 & 14 \\
\hline
\end{tabular}

Migraine migraine without aura, $C G H$ cervicogenic headache, $M H F$ multiple headache forms

20 subjects with MHF (15 female, mean age 33 years, range 20-63, SD 9.4) were recruited. Headache symptoms and other characteristics are shown in Table 1.

\section{Procedures}

Entry-level eligibility was based on inclusion and exclusion criteria and assessed by a separate researcher, an experienced specialist musculoskeletal physiotherapist. Subsequently all subjects completed a questionnaire to obtain an index of headache severity (0-100, with 100 being maximum severity) [30]. This index is based on a composite score of headache frequency, duration, and intensity with equal weight given to each element. Previously this index has been shown to have high levels of reliability with $\operatorname{ICC}_{(2,1)}$ of 0.92 [30]. Additional demographic details, including subject and headache characteristics were also assessed by questionnaire. These characteristics included: dominant side of headache, headache of pulsating quality, nausea, photophobia or phonophobia, anti-migraine medication helps, forward trunk bend increases pain, neck movement or positions provoke headache, headache aggravated by exertion, and neck symptoms precede headache.
One specialist musculoskeletal physiotherapist experienced in using the FRT, who was unaware of the subject's clinical presentation, assessed all subjects. An experienced examiner was utilized, as the purpose of this study was not to investigate reliability but to investigate differences in impairment of range of motion during the FRT in subjects with different headache forms. We have previously established high levels of intra-rater reliability for range of motion measured by an experienced examiner. Intra-class correlation coefficients were reported as 0.95 (95\% CI 0.90-0.98) [23]. Furthermore, examiner interpretation of the FRT has been shown to be consistent over time with Kappa of 0.92 [23].

The FRT consisted of pre-positioning the cervical spine in maximal end range flexion followed by passive rotation of the head to the left and the right, with the subject relaxed in supine. End of range was determined either by firm resistance encountered by the therapist or the subject reporting the onset of pain, whichever came first. The intention was to measure range of motion irrespective of cause of limitation, in the least provocative manner, to prevent potential exacerbation of symptoms. The examiner made a visual estimate of the range of rotation to determine whether the FRT was positive or negative, and then reported the test state and the positive direction if any. 
A positive test was defined where the visually estimated range was reduced by more than $10^{\circ}$ from the anticipated normal range of $44^{\circ}[21,24]$. Subsequently the FRT procedure was repeated and mobility determined by using a cervical range of motion device (CROM). The CROM is a floating compass [Plastimo Airguide Inc (Compasses), 1110 Lake Cook Road, Buffalo Groove, Illinois, 60089] attached to the apex of the head by Velcro straps [21, 24].

All subjects were tested on one occasion on a headachefree day to negate the influence of headache at the time of testing [23]. All 11 subjects complained of headache on the day of testing and were required to return on a symptomfree day.

\section{Statistical analysis}

Statistical analysis was carried out using SPSS V17.0. (SPSS Inc., 444 N. Michigan Avenue, Chicago, Illinois, 60611). Alpha was set at 0.05 for each analysis. One-way ANOVA and planned orthogonal comparisons were used to determine differences in FRT mobility between groups. The sensitivity and specificity of the FRT were analysed using cross tabulation and were determined with a receiver operating characteristic (ROC) curve. To calculate the sensitivity and specificity the subjects with Migraine and MHF were combined and then compared to the subjects with CGH. The frequencies used to calculate sensitivity and specificity are given in Table 1 . The dichotomous variables used to determine the sensitivity and specificity were the therapist's assessment of whether the FRT was positive $\left(10^{\circ}\right.$ visually estimated limitation of range $)$ and whether the subject had CGH or not. The ROC curve was created using the FRT range of motion values. Finally, forward stepwise multiple linear regression analysis (forward stepwise; $P$ to enter $<0.05, P$ to remove $>0.1$ ) was used to determine the relationship between subject and headache characteristics and range of motion during the FRT.

\section{Findings}

Subject recruitment occurred from January to December 2008. All 60 subjects completed this study. The demographic and headache characteristics of the study sample are presented in Table 1.

All underlying assumptions for repeated measure ANOVA were met. The range of rotation to the most restricted side was significantly lower in the CGH group when compared to the Migraine and MHF groups $\left[P \leq 0.001\right.$; mean $(\mathrm{SD})$ range $25.2^{\circ}(11.1), 41.8^{\circ}(5.1)$ and $35.1^{\circ}$ (8.0), respectively] (Fig. 1). There was also a

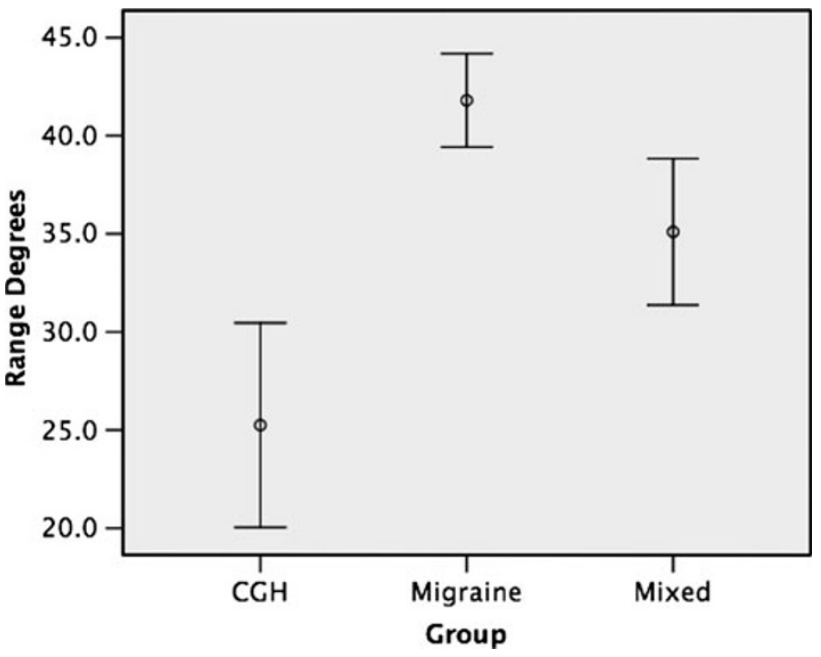

Fig. 1 The mean range of motion and 95\% confidence interval of unilateral cervical rotation in maximal flexion to the most restricted side for each subject group

significant but smaller difference in range between the Migraine and MHF groups $(P=0.039)$ (Fig. 1).

The examiner interpreted the FRT as positive in $70 \%$ of subjects (14/20) from the CGH group and 30\% of subjects (12/40) from the combined groups Migraine and MHF. Consequently for CGH diagnosis the FRT had sensitivity of 0.7 (95\% CI 0.46-0.87), specificity of 0.7 (95\% CI $0.53-$ 0.83 ), positive predictive value of 0.54 (95\% CI 0.34 $0.74)$, negative predictive value of $0.82(95 \%$ CI $0.65-0.93$ ), positive likelihood ratio of 2.33 (95\% CI 1.34 4.06) and negative likelihood ratio of 0.43 (95\% CI $0.21-$ 0.85). Examination of sensitivity and specificity data plotted on a ROC curve (Fig. 2) revealed that an experienced clinician should be able to correctly differentiate a patient with $\mathrm{CGH}$ from one from the Migraine or MHF groups $85 \%$ of the time $(P<0.001)$. Additionally, coordinates on the ROC curve indicated that, in this sample, the test value that provides the highest sensitivity and the lowest 1 -specificity was $30^{\circ}$. In other words range of $30^{\circ}$, measured using a compass goniometer, represents the cutoff score for a positive test.

When variables "neck movement or positions provoke headache" and "neck symptoms precede headache" and other variables (gender, age, history of headache, dominant side of headache, anti-migraine medication helps, forward bend increases headache, presence of photophobia or phonophobia, nausea or headache of pulsating quality) were entered into forward stepwise multiple linear regression analysis with range of motion towards the most restricted side during the FRT as the dependent variable, "neck movement or positions provoke headache" and "neck symptoms precede headache" were the most significant predictors of range of motion (Table 2). These 


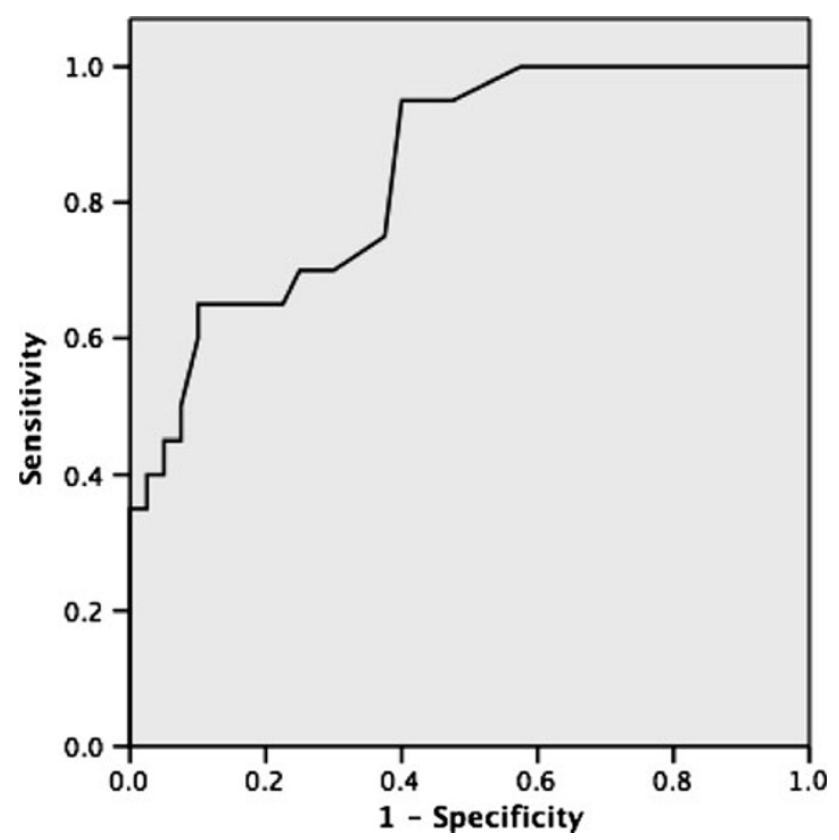

Fig. 2 The diagnostic accuracy of the cervical flexion-rotation test. The area under the curve is $0.85(P<0.001$, CI $0.75,0.95)$

Table 2 Forward stepwise multiple linear regression analysis with $\beta$, standard error, $P$, and adjusted $R^{2}$ values presented: predictive variables for range of motion towards the most restricted side during the flexion-rotation test (FRT) $(n=60)$

\begin{tabular}{llll}
\hline Variable & $\begin{array}{l}\text { Coefficient } \\
(\beta)\end{array}$ & $\begin{array}{l}\text { Standard } \\
\text { error }\end{array}$ & \\
\hline Constant & 10.17 & 3.64 & $<0.001$ \\
$\begin{array}{l}\text { Neck positions/movement provoke } \\
\text { headache }\end{array}$ & 10.83 & 2.55 & $<0.001$ \\
Neck symptoms precede headache & 5.51 & 2.54 & 0.04 \\
\hline
\end{tabular}

Adjusted $R^{2}=0.44, R^{2}$ change for "Neck positions/movement provoke headache" $=0.41, R^{2}$ change for "Neck symptoms precede headache" $=0.04$. Variables entered forward stepwise

variables accounted for $44 \%$ of the variance in FRT range of motion $[F(2,59)=24.5, P<0.001]$, with neck movement or positions provoke headache as the most significant predictor accounting for $41 \%$ of the variance. Once these two variables had been entered into the model, none of the remaining independent variables made sufficient additional contribution to the model to meet the entry criterion.

\section{Discussion}

This is the first reported study to compare FRT range of motion deficits in subjects with CGH, Migraine and MHF. We found a clinically significant difference in range of motion between the different headache groups. Range was most restricted in subjects with $\mathrm{CGH}\left(25^{\circ}\right)$, significantly greater impairment than either group's Migraine $\left(42^{\circ}\right)$ or MHF $\left(35^{\circ}\right)$. Similarly, subjects with MHF had significantly lesser range than those subjects with Migraine. The data for range of motion measured during the FRT are similar to those reported in previous studies using the CROM device in subjects with $\mathrm{CGH}[26,31]$. Mean range of rotation towards the most restricted side was $25^{\circ}$ in the current study and $22^{\circ}$ and $26^{\circ}$ in previous reports [26, 31]. In the present study an asymptomatic group was not included, but compared to previous studies range recorded in the Migraine group was marginally less than the reported normal range of $44^{\circ}-45^{\circ}$ to each side $[21,32]$.

Our results for mean range of motion for subjects with Migraine concur with a previous report of mean range of $39^{\circ}$ for subjects with migraine with aura [24]. It would appear that the presence of an aura has minimal effect on range of motion during the FRT. As range of motion for subjects with Migraine was also consistent with asymptomatic controls this might indicate that, in general terms, cervical movement dysfunction is not a pre-requisite for migraine either with or without aura. This observation is consistent with a previous study investigating active range of motion. Subjects with Migraine had range consistent with asymptomatic controls, which was significantly greater than range in subjects with $\mathrm{CGH}[19,33]$. In another study of episodic migraine, only range of right rotation was significantly reduced by $8^{\circ}$ when compared to healthy controls [34]. Interestingly in cases of unilateral pain, limitation was not associated with the symptomatic side of migraine [34], which is different from CGH where the limitation of movement is predominantly to the symptomatic side, particularly when evaluated by the FRT. Further evidence of a lack of cervical movement impairment in Migraine was demonstrated by a recent systematic review, although the reviewers were critical of the methodological quality of many of the reviewed studies [35].

Migraine headache is experienced in the regions innervated by the cutaneous afferents of the trigeminal and $\mathrm{C} 2$ and $\mathrm{C} 3$ nerves, all of which have input into the TCN [35]. Sensitization of the TCN is postulated as central to the pathophysiology of $\mathrm{CGH}$, migraine and other headache forms and may explain why some patients with headache complain of neck pain [12], and demonstrate limitation of cervical range of motion, in the absence of cervical musculoskeletal disease [36]. Furthermore sensitization of the TCN may also increase neck muscle tone through increased motor efferent activity, with consequent effect of limiting range of motion of the neck [37]. If TCN sensitization were the cause of headache then limitation in range is unlikely to be related to a specific side or level of cervical motion segment dysfunction, and the FRT is unlikely to be significantly restricted. We found only $5 / 20$ subjects with migraine with a positive FRT, indicating a lack of 
segmental joint restriction in the majority of migraine sufferers. Consistent with this and as previously stated no association was found between the side of migraine headache and direction of movement limitation [34]. Thus for a patient with headache, associated neck pain and minor limitation of cervical range of motion does not necessarily indicate a cervical contributing factor. Further physical examination tests are required in that patient to confirm the presence of CGH. It is the combination of limitation of cervical movement together with evidence of deep neck flexor dysfunction and pain on palpation of the upper cervical spine that confirms the presence of CGH with a high degree of certainty [20].

Previously it has been shown that the FRT has good diagnostic accuracy to identify subjects with CGH from subjects with migraine with aura and asymptomatic controls [24, 26]. In the study by Ogince et al. [24] subjects with CGH were compared with control subjects, whereas in this study the comparison was with subjects with Migraine or MHF. This difference in comparison groups would also explain the slightly lower cut-off value in the present study when compared with earlier studies $\left(30^{\circ}\right.$ against $\left.32^{\circ}\right)$ [26]. This has implications for clinical practice where diagnosis of CGH might rely on a number of features including presenting symptoms as well as physical examination [22]. In patients who have subjective features that do not fit into a specific headache category (or who have evidence of MHF) then the physical examination might be more important and a lower cut-off score is necessary on the FRT to be confident of a diagnosis of CGH. In patients with relatively pure form of $\mathrm{CGH}$, clearly defined by the presenting symptoms, then a higher cut-off score might be adequate.

It is important to recognise that the FRT is a relatively isolated test of movement impairment of the C1/2 motion segment [25], and may not adequately test other motion segments or indeed other upper cervical structures potentially contributing to a patient's CGH. This might explain the negative test finding in $6 / 20$ subjects with $\mathrm{CGH}$, where cervical motion segments or structures other than $\mathrm{C} 1 / 2$ may have been the cause of headache. Hence it is important not to rule out the potential for CGH in patients negative on the FRT.

We found that variables forward bend increases headache, anti-migraine medication helps, photophobia, phonophobia, nausea, pulsating headache, exertion increases headache, history of headache, gender, and age were not associated with range of motion towards the restricted side during the FRT. The only factors associated with range of motion were neck movement or positions provoke headache, and neck symptoms precede headache. The variable neck position or movement provoke headache accounts for $41 \%$ of the variance (adjusted $R^{2}=0.41$ ). The inclusion of the variable neck pain precedes headache results in an additional $4 \%$ of the variance $\left(R^{2}=0.4\right)$. This result is not surprising when considering these two associated factors are determinants of $\mathrm{CGH}$ and that apart from age, gender and history of headache, the non-associated factors are common complaints of migraine, but rarely reported by subjects with CGH [38]. Previously we have also shown that three headache features (headache severity, frequency and duration), either separately or combined into an index [30], also influence range of motion during the FRT in subjects with CGH [31]. In addition we have shown that the presence of pain at the time of testing also influences range of motion [23]. Hence the FRT appears to be associated with a number of different factors including the presence and severity of headache as well as the factors suggesting cervical spine dysfunction.

The interpretation and generalization of these results needs to be cautioned by a number of potential limitations. Firstly, one experienced examiner tested all subjects. Secondly a diagnosis of "probable" CGH was only possible as diagnostic injection block procedures were not available, hence some subjects may have been misclassified. Finally 14/20 subjects with CGH were positive on the FRT, which may indicate that subjects were misclassified as CGH or did not have headache arising from $\mathrm{C} 1 / 2$.

\section{Conclusions}

Range of motion towards the most restricted side during the FRT is significantly reduced in subjects with CGH when compared to subjects with Migraine or MHF. Furthermore, the FRT has good sensitivity and specificity in the diagnosis of CGH and the cut-off value for a positive test is range of motion less than $30^{\circ}$ for differentiation between these headache groups. Subjective features characteristic of Migraine are not associated with FRT range of motion but features consistent with CGH are. These findings provide further evidence supporting the clinical utility of the FRT.

Conflict of interest None.

\section{References}

1. Stovner L, Hagen K, Jensen R, Katsarava Z, Lipton R, Scher A, Steiner T, Zwart J (2007) The global burden of headache: a documentation of headache prevalence and disability worldwide. Cephalalgia 27:193-210

2. Diener I (2001) The impact of cervicogenic headache on patients attending a private physiotherapy practice in Cape Town. S Afr J Physiother 57(1):35-39

3. Lipton RB, Stewart WF, Diamond S, Diamond ML, Reed M (2001) Prevalence and burden of migraine in the United States: 
data from the American Migraine Study II. Headache 41(7):646657

4. Lipton RB, Bigal ME, Diamond M, Freitag F, Reed ML, Stewart WF (2007) Migraine prevalence, disease burden, and the need for preventive therapy. Neurology 68(5):343-349

5. Buzzi M, Moskowitz M (2005) The pathophysiology of migraine: year 2005. J Headache Pain 6:105-111

6. Aurora SK, Barrodale PM, Tipton RL, Khodavirdi A (2007) Brainstem dysfunction in chronic migraine as evidenced by neurophysiological and positron emission tomography studies. Headache 47(7):996-1003 (discussion 1004-1007)

7. Jull G (2002) Management of cervicogenic headache. In: Grant R (ed) Physical therapy of the cervical and thoracic spine. Churchill-Livingstone, New York, pp 239-272

8. Bogduk N, Govind J (2009) Cervicogenic headache: an assessment of the evidence on clinical diagnosis, invasive tests, and treatment. Lancet Neurol 8(10):959-968

9. Dodick DW (2010) Pearls: Headache. Semin Neurol 30(1):74-81

10. Classification Committee of the International Headache Society (2004). The international classification of headache disorders, 2nd edn. Cephalalgia 24(Suppl 1):9-160

11. Sjaastad O, Fredriksen TA, Pfaffenrath V (1998) Cervicogenic headache: diagnostic criteria. The Cervicogenic Headache International Study Group. Headache 38(6):442-445

12. Calhoun AH, Ford S, Millen C, Finkel AG, Truong Y, Nie Y (2010). The Prevalence of Neck Pain in Migraine. Headache

13. Henry P, Dartigues J, Puymirat C, Peytour P, Lucas J (1987) The association cervicalgia-headaches: an epidemiologic study. Cephalalgia 7(Suppl. 6):189-190

14. Pfaffenrath V, Kaube H (1990) Diagnostics of cervicogenic headache. Funct Neurol 5(2):159-164

15. Hall T, Ho Tak Chan B, Christensen L, Odenthal B, Wells C, Robinson K (2007) Efficacy of a C1/2 self-SNAG (sustained natural apophyseal glide) in the management of cervicogenic headache. J Orthop Sports Phys Ther 37(3):100-107

16. Jull G, Trott P, Potter H, Zito G, Neire K, Shirley D, Emberson J, Marschner I, Richardson C (2002) A randomized controlled trial of exercise and manipulative therapy for cervicogenic headache. Spine 27(17):1835-1843

17. Bronfort G, Nilsson N, Haas M, Evans R, Goldsmith $\mathrm{CH}$, Assendelft WJ, Bouter LM (2004). Non-invasive physical treatments for chronic/recurrent headache. Cochrane Database Syst Rev (3):CD001878

18. Blau J, MacGregor E (1994) Migraine and the neck. Headache 34:88-90

19. Zito G, Jull G, Story I (2006) Clinical tests of musculoskeletal dysfunction in the diagnosis of cervicogenic headache. Man Ther 11(2): $118-129$

20. Jull G, Amiri M, Bullock-Saxton J, Darnell R, Lander C (2007) Cervical musculoskeletal impairment in frequent intermittent headache. Part 1: Subjects with single headaches. Cephalalgia 27(7):793-802
21. Hall T, Robinson K (2004) The flexion-rotation test and active cervical mobility - a comparative measurement study in cervicogenic headache. Man Ther 9(4):197-202

22. Hall TM, Briffa K, Hopper D (2008) Clinical evaluation of cervicogenic headache. J Man Manip Ther 16(2):73-80

23. Hall TM, Briffa K, Hopper D, Robinson KW (2010) Intratester reliability and minimal detectable change of the cervical flexionrotation test. J Orthop Sports Phys Ther 40(4):225-229

24. Ogince M, Hall T, Robinson K, Blackmore AM (2007) The diagnostic validity of the cervical flexion-rotation test in $\mathrm{C} 1 / 2$ related cervicogenic headache. Man Ther 12(3):256-262

25. Takasaki H, Hall T, Oshiro S, Kaneko S, Ikemoto Y, Jull G (2010). Normal kinematics of the upper cervical spine during the flexion-rotation test-in vivo measurements using magnetic resonance imaging. (submitted)

26. Hall TM, Robinson KW, Fujinawa O, Akasaka K, Pyne EA (2008) Intertester reliability and diagnostic validity of the cervical flexion-rotation test. J Manip Physiol Ther 31(4):293-300

27. Eliasziw M, Young S, Woodbury M, Fryday-Field K (1994) Statistical methodology for the concurrent assessment of interrater and intrarater reliability: using goniometric measurements as an example. Phys Ther 74:777-788

28. Faul F, Erdfelder E, Lang AG, Buchner A (2007) G*Power 3: a flexible statistical power analysis program for the social, behavioral, and biomedical sciences. Behav Res Methods 39(2):175-191

29. Antonaci F, Ghirmai S, Bono G, Sandrini G, Nappi G (2001) Cervicogenic headache: evaluation of the original diagnostic criteria. Cephalalgia 21(5):573-583

30. Niere K, Robinson P (1997) Determination of manipulative physiotherapy treatment outcome in headache patients. Man Ther 2(4):199-205

31. Hall TM, Briffa K, Hopper D (2010). The relationship between cervicogenic headache subjective features and impairment determined by the cervical flexion-rotation test. (submitted)

32. Smith K, Hall T, Robinson K (2007) The influence of age, gender, lifestyle factors and sub-clinical neck pain on cervical range of motion. Man Ther 13:552-559

33. Zwart JA (1997) Neck mobility in different headache disorders. Headache 37(1):6-11

34. Bevilaqua-Grossi D, Pegoretti KS, Goncalves MC, Speciali JG, Bordini CA, Bigal ME (2009) Cervical mobility in women with migraine. Headache 49(5):726-731

35. Robertson BA, Morris ME (2008) The role of cervical dysfunction in migraine: a systematic review. Cephalalgia 28(5):474-483

36. Niere K (2009) Central nervous system processing in cervicogenic headache. In: Selvaratnam PJ, Niere K, Zuluaga M (eds) Headache orofacial pain and bruxism. Elsevier, Edinburgh, pp 95-114

37. Bartsch T (2005) Migraine and the neck: new insights from basic data. Curr Pain Headache Rep 9(3):191-196

38. Sjaastad O, Bakketeig LS (2007) Migraine without aura: comparison with cervicogenic headache Vaga study of headache epidemiology. Acta Neurol Scand 117(6):377-383 